\title{
Turning a Graphics Tablet into a Transparent Blackboard
}

\author{
Jörn Loviscach* \\ Fachhochschule Bielefeld (University of Applied Sciences)
}

\section{Introduction}

Most lectures in mathematics and in the natural sciences make prominent use of blackboards. Developing an idea is much more natural and interactive with hand-drawn sketches than with glitzy prepared slides. The art of drawing is undergoing a renaissance, which can be seen in literature [Roam 2008] as well as in educational videos on the Web. This work demonstrates a low-cost realtime system to visually enhance such lectures. Unlike related past approaches that started with [Ishii and Kobayashi 1992], it works with a minimal setup such as a tablet PC with integrated webcam.

A regular blackboard and a regular sheet of paper lack visual clarity as opposed to a computer's crisp output. Hence, it makes sense to use a graphics tablet or a Tablet PC in conjunction with appropriate drawing and writing software. This offers the benefit of digital storage, endless scrolling, and editing operations such as undo, cut/copy/paste, resize, rotate as well as precise and residue-free delete. Handwriting recognition enables automated indexing. Even though this recognition is of limited reliability, it is highly superior to recognizing writing on a filmed regular blackboard.

However, lectures presented on a graphics tablet and the pencasts produced from them suffer from two problems: First, the hand of the presenter is not visible. This makes it harder to follow the writing and pointing, even though the tip of the digital pen may be highlighted on the screen. Second, the presenter's talking head is not visible in close vicinity to the writing. Sometimes the head is added in a distracting fashion as a separate, second video stream.

\section{Techniques}

To address these issues, this work proposes a novel style and a novel technique for pencasts: The input from a regular graphics tablet or tablet PC controls an animated model of a writing hand. A webcam captures the face of the presenter. These graphical ingredients are composed to create the illusion of the presenter writing on the back of a transparent board, see Fig. 1. This style is inspired by the visuals of science fiction movies-which themselves borrow heavily from the plotting boards of historic fighter-control centers-as well as Hollywood's depictions of mathematics nerds such as the main character of "A Beautiful Mind," who scribbles on windows. Color controls allow picking a highly subdued look for the face and the hand so that they convey just enough information. The hand is behind the board so that it never occludes the writing.

The illusion of a transparent board is sold through reflections on the imaginary surface and through a grid of guidelines that scroll with the blackboard's content. A soft glow below the tip indicates if the stylus touches the surface and shows the current drawing color.

The hand and arm are 2D images animated through inverse kinematics driven by the motion of the stylus. When the stylus leaves the detection range of the graphics tablet, the tip's position is no longer known and the system resorts to letting the arm drop out of the image with constant acceleration. When the presenter turns the stylus around to switch to the eraser tool, the image of the hand changes accordingly. For instance, the palm's hypothenar can be highlighted when it is used for erasing.

*e-mail: joern.loviscach@fh-bielefeld.de

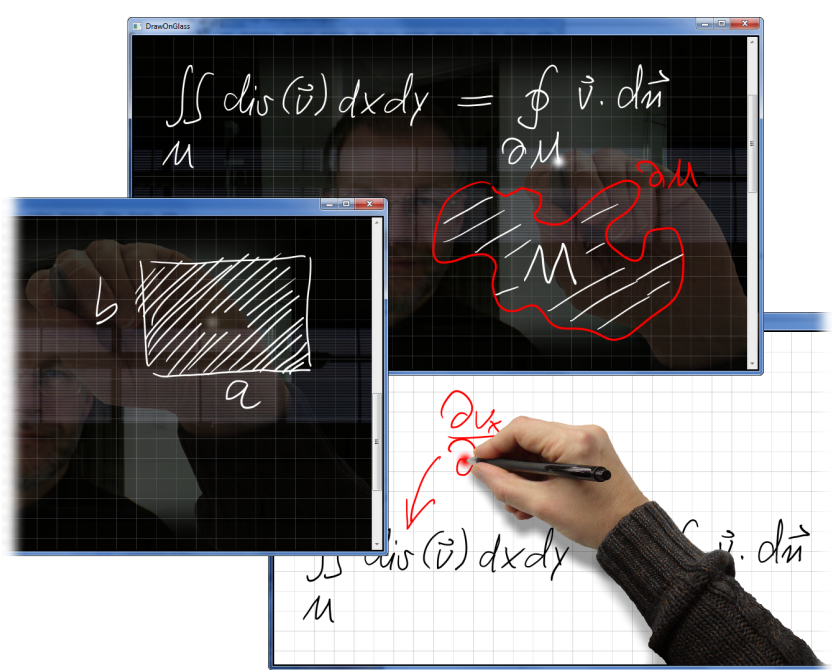

Figure 1: A graphics tablet and a webcam emulate a transparent blackboard; additional styles include handwriting on paper.

People are used to looking at mirrors, but not to interact with truesided images of themselves. Hence, to not confuse the presenter, the hand and the face are shown horizontally flipped. Note that an actual transparent blackboard requires writing in mirror script.

Stereo sound is synthesized from the velocity, pressure, and location data of the graphics tablet. These control volume, pitch, and stereo position in real time. The sound is built from two layers of long seamless loops created from actual audio recordings of pen writing. The pen tool has a hissing base sound to which a squeaking sound is mixed if the pressure is high. For the eraser tool, only the squeaking sound is used. These sounds indicate when and where writing is going on with which intensity.

\section{Outlook}

Future work will implement further styles. A second mode already simulates a hand drawing on a white background as found in the highly popular RSA Animates by Cognitive Media [RSA 2011]. A ruler to draw lines and a compass to draw circles can be added. A multitouch panel may be used to show both hands or even to animate several fingers independently, which is of particular interest for rear-touch displays such as [Wigdor et al. 2007].

\section{References}

IshII, H., AND KoBAYASHI, M. 1992. ClearBoard: a seamless medium for shared drawing and conversation with eye contact. In $\mathrm{CHI}$ '92, 525-532.

Roam, D. 2008. The Back of the Napkin: Solving Problems and Selling Ideas with Pictures. Portfolio, Penguin, NY.

RSA, 2011. RSA Animates. http://comment.rsablogs. org.uk/videos/.

Wigdor, D., Forlines, C., Baudisch, P., BARnwell, J., AND SHEN, C. 2007. Lucid touch: a see-through mobile device. In UIST '07, 269-278. 